\title{
A fully automated scanning ratio calorimeter for use in adsorption studies
}

\author{
Mark Lysek, ${ }^{\text {a) }}$ Peter Day, Marissa LaMadrid, and David Goodstein \\ Condensed Matter Physics 114-36, California Institute of Technology Pasadena, California 91125
}

(Received 27 April 1992; accepted for publication 23 July 1992)

\begin{abstract}
This article describes a scanning ratio calorimeter that has been developed for studying multilayer adsorbed films. The scanning technique presented is able to accumulate highly precise data much more quickly than other calorimetric techniques. The rms noise in the total heat capacity of the sample cell is $0.02 \%$ at $70 \mathrm{~K}$ and $0.04 \%$ at $120 \mathrm{~K}$ for scan rates of $2 \mathrm{~K} / \mathrm{h}$. Sources of error in the heat capacity measurement are analyzed in detail. The calorimeter is completely automated and can be operated continuously using a personal computer.
\end{abstract}

\section{INTRODUCTION}

Heat capacity measurements are probably the most sensitive means to identify when a substance changes phase, particularly in an adsorbed film. Most other experimental techniques measure a material property of a substance, e.g., the lattice constant. When there is a phase transition, the material property changes either discontinuously or smoothly, but the heat capacity usually changes sharply. It is far easier to locate a peak in real experimental data than a small discontinuity or change of slope on a smoothly changing background. For example, in adsorbed systems vapor pressure isotherms typically exhibit a near discontinuity in the density as a function of pressure when the system passes through a first-order transition, but the heat capacity records a well-defined peak. Whenever a phase change occurs in any system, there is a discontinuity in either the entropy or its $N$ th derivative with respect to temperature at constant pressure. ${ }^{1}$ Since the heat capacity is proportional to the first derivative of the entropy, $C$ $=T d S / d T$, it is especially sensitive to changes of phase. However, the heat capacity of the sample cell and adsorption substrate combined (the background heat capacity) is typically 10-100 times larger than that of the film. A measurement of the total sample cell heat capacity (background plus film) accurate to $0.02 \%$ translates to an accuracy of $0.2 \%-2.0 \%$ for the film heat capacity alone. Thus, very sensitive heat capacity measurements are required.

Previous heat capacity studies of monolayer and multilayer films have used either discrete pulse calorimetry or high precision ac calorimetry. In the discrete pulse method, a predetermined amount of heat is put into the sample cell, and the change in temperature is measured after equilibrium is reestablished. Because it is impossible to maintain the sample's environment at the sample temperature while it is changing rapidly, an unknown amount of heat is exchanged between the sample and its environment during the measurement. For this reason, discrete pulse techniques are generally an order of magnitude less sensitive than the one we will describe.

In ac calorimetry, the sample heater power is varied sinusoidally and the thermal environment is kept at fixed

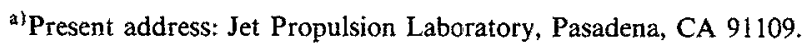

temperature. The heat capacity is derived from the temperature variation of the sample cell, and corrections due to the thermal link between the sample and its environment can be subtracted. The advantage of this technique is that elaborate thermal isolation for the cell is unnecessary. Long integration times can give very high precision data. A disadvantage of the ac method is that the temperature must be stabilized at each point at which data are to be collected. Added to the necessity of long integration times, each data point requires a considerable time investment.

Scanning techniques can achieve the sensitivity of ac techniques, but accumulate data much more quickly. Figure 1 is an illustration of a scanning ratio calorimeter. In scanning calorimetry, a comparison thermal mass (CTM) with a smooth heat capacity is heated at a constant rate, resulting in a nearly linear temperature rise. Meanwhile, the sample heat input is adjusted to keep the temperature difference between cell and CTM equal to zero. The two bodies are surrounded by a temperature controlled radiation shield to reduce the stray heat flow. The heat capacity of the sample is then the ratio of the sample heater power to the CTM heater power multiplied by the CTM heat capacity. As we will discuss below, it is possible to achieve a sensitivity of a few parts in $10^{4}$ with this technique.

The advantage of scanning calorimetry is that using moderate scan rates, large quantities of data can be accumulated in less time. The scan rate must be slow enough to ensure that the sample is close to equilibrium at all times. Nonequilibrium effects can be easily checked for by varying the scan rate. Slow scan rates improve the temperature resolution, approximately the scan rate multiplied by the thermal relaxation time of the sample. Very low scan rates are also undesirable because the effect of stray heat leaks varies inversely as the scan rate. We have chosen a scan rate of $2 \mathrm{~K} / \mathrm{h}$ for most of our work (temperature range 60 $\mathrm{K} \leqslant T \leqslant 100 \mathrm{~K}$ ) to ensure that the sample is close to equilibrium while providing adequate noise performance. The resulting temperature resolution is about $0.1 \mathrm{~K}$. By using slower scan rates, increased temperature resolution may be obtained at the expense of the calorimetric accuracy.

Figure 2 shows several heat capacity scans taken with the calorimeter we describe in this article. Different curves correspond to different film coverages between 0.87 and 5.27 layers. The sensitivity of the instrument allows the identification of many previously unobserved features in 


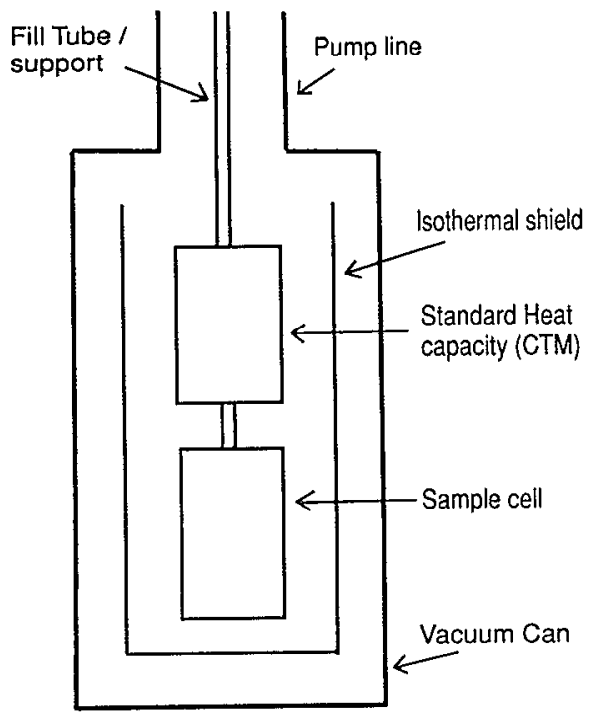

FIG. 1. A scanning ratio calorimeter.

the data. More data taken with this instrument may be found in Refs. 2, 3.

In Sec. II of this article, we will examine the requirements for measuring heat capacities accurate to $\sim 0.01 \%$ of the background using a scanning technique. Section III describes the design actually implemented for studying multilayer films where we achieve a sensitivity of $0.02 \%$ $0.04 \%$. In Sec. IV, we analyze the noise in data taken with our instrument.

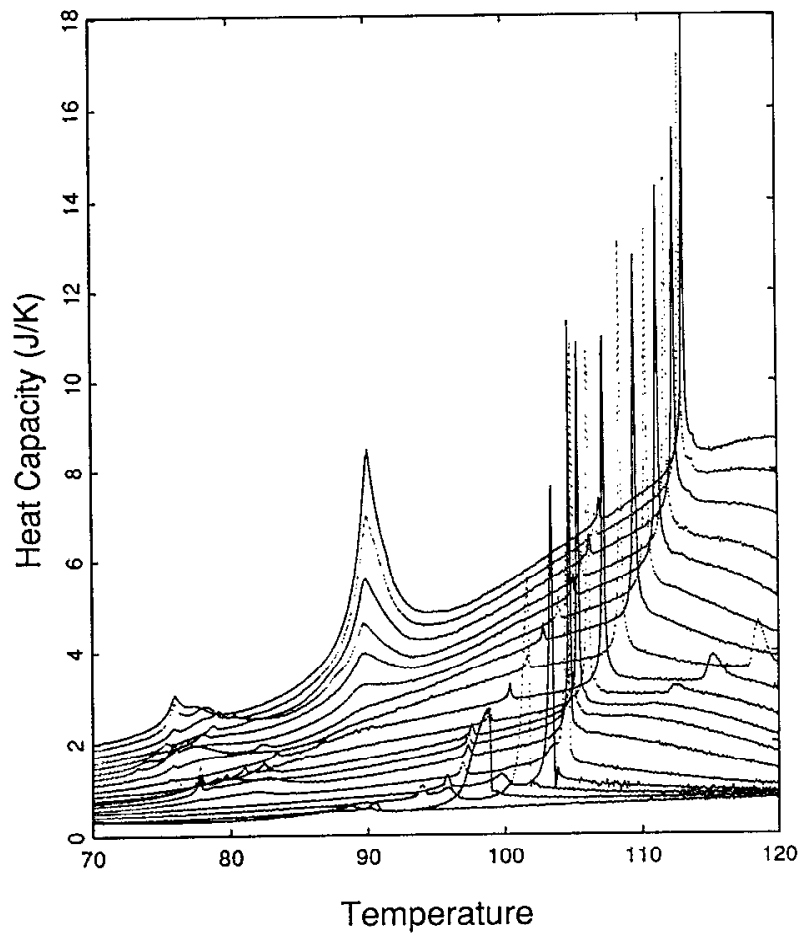

FIG. 2. Heat capacity scans for methane on graphite. Different curves correspond to different coverages between 0.87 and 5.27 monolayers.

\section{DESIGN REQUIREMENTS}

The configuration we will analyze below compares two bodies. Similar methods were used by many experimenters, e.g., Refs. 4 and 5 . The first body holds the sample, having heat capacity $C_{0}$, temperature $T_{0}$, heat input $W_{0}$, and unknown heat leak $W_{x 0}$. The second has known heat capacity $C_{1}$, temperature $T_{1}$, heat input $W_{1}$, and unknown heat leak $W_{x 1}$. The two cells are coupled together with a weak thermal coupling $K$, so that $K\left(T_{1}-T_{0}\right)$ Watts flow into the sample cell. Then the first-order differential equations for both bodies are

$$
\begin{aligned}
& \frac{d T_{0}}{d t}=\frac{W_{0}+K\left(T_{1}-T_{0}\right)-W_{x 0}}{C_{0}\left(T_{0}\right)}, \\
& \frac{d T_{1}}{d t}=\frac{W_{1}-K\left(T_{1}-T_{0}\right)-W_{x 1}}{C_{1}\left(T_{1}\right)} .
\end{aligned}
$$

By subtracting these two equations, an expression for $C_{0}(T)$ may be obtained in terms of $T_{0}, C_{1}(T)$, and the temperature difference $\delta T=T_{0}-T_{1}$. To find the film heat capacity we must subtract the heat capacity of the empty cell and bare graphite substrate, $C_{0}^{\prime}(T)$, measured during a separate background run:

$$
\begin{aligned}
C_{\text {film }}\left(T_{0}\right)= & C_{0}\left(T_{0}\right)-C_{0}^{\prime}\left(T_{0}\right) \\
= & \frac{C_{1}\left(T_{1}\right)\left(W_{0}-K \delta T-W_{x 0}\right)}{C_{1}\left(T_{1}\right) \frac{d \delta T}{d t}+W_{1}+K \delta T-W_{x 1}} \\
& -\frac{C_{1}^{\prime}\left(T_{1}^{\prime}\right)\left(W_{0}^{\prime}-K^{\prime} \delta T^{\prime}-W_{x 0}^{\prime}\right)}{C_{1}^{\prime}\left(T_{1}^{\prime}\right) \frac{d \delta T^{\prime}}{d t}+W_{1}^{\prime}+K^{\prime} \delta T^{\prime}-W_{x 1}^{\prime}} .
\end{aligned}
$$

Note that $T_{1}^{\prime}=T_{0}^{\prime}-\delta T^{\prime}$ is not necessarily equal to $T_{1}$. It is useful to linearize with respect to the small quantities $C_{1} d \delta T / d t, K \delta T, W_{x 0}$, and $W_{x 1}$. To first order in these:

$$
C_{\text {film }}=F+G+H+L,
$$

where

$$
\begin{aligned}
& F=C_{1} \frac{W_{0}-W_{0}^{\prime}}{W_{1}}, \\
& G=\frac{1}{D^{2}}\left(W_{0} \frac{d \delta T}{d t}-W_{0}^{\prime} \frac{d \delta T^{\prime}}{d t}\right), \\
& H=\frac{1}{D}\left[\left(1+\frac{W_{0}}{W_{1}}\right) K \delta T-\left(1+\frac{W_{0}^{\prime}}{W_{1}^{\prime}}\right) K^{\prime} \delta T^{\prime}\right], \\
& L=\frac{1}{D}\left[\left(W_{x 0}-W_{x 1} \frac{W_{0}}{W_{1}}\right)-\left(W_{x 0}^{\prime}-W_{x 1}^{\prime} \frac{W_{0}^{\prime}}{W_{1}^{\prime}}\right)\right],
\end{aligned}
$$

and $W_{1} / C_{1}=W_{1}^{\prime} / C_{1}^{\prime}=D$, the CTM drift rate per unit time. Here we assumed that the background run was taken with the same CTM heat capacity and heater power, $C_{1}^{\prime}=C_{1}$ and $W_{1}^{\prime}=W_{1}$. Below, we will examine each term.

$F$ is the ideal value for the film heat capacity assuming that the temperature control is perfect and there are no heat leaks. $G$ is the correction for the small difference in the 
sample cell and CTM drift rates. As the cell temperature drifts through features in the heat capacity, there will be some small error in the temperature control. This can be corrected for by numerically differentiating the $\delta T$ data. The statistical and systematic errors in computing this derivative are significant and will be discussed below. $H$ represents the error caused by the finite thermal coupling between the two bodies and the errors in the sample temperature control. With accurate temperature control, and careful design to keep the coupling low, this term can be made small. All the stray heat leaks into the cell and CTM are lumped into $L$. There are two sources of such heat leaks. The first is thermal contact (gaseous heat conduction and thermal radiation) between each body and the radiation shield. The second is heat flow through the bodies' mechanical supports including the gas supply lines. Note that if the background run has similar heat leaks, there will be some cancellation in the expression for $L$. A major purpose in the design of the calorimeter is to reduce the size of this term.

The size of each term above determines the requirements for a part of the probe and associated instruments. We will first discuss the rough technical requirements for making each term's error contribution near the desired accuracy of $0.01 \%$, then in the sections that follow we will describe the details of the instruments actually used.

\section{A. Errors in the ideal film heat capacity}

By far the largest term in the expression for the film heat capacity is $F$, simply the ratio of the cell to CTM power inputs multiplied by the CTM heat capacity. The CTM heat capacity is computed from the relationship:

$$
C_{\mathrm{std}}=W_{\mathrm{std}} / \frac{d T_{\mathrm{std}}}{d t}
$$

using data from the background heat capacity run. The CTM heat capacity may be accurately fit to a low-order polynomial to reduce the large statistical errors caused by computing the derivative numerically. Systematic errors in this measurement are caused by errors in the heater power and errors in the temperature scale for the thermometer.

The thermometry errors were made as small as practical by purchasing a miniature platinum thermometer calibrated against a secondary standard by Lake Shore Cryotronics. $^{6}$ Strain-free platinum thermometers and certain fixed temperature points define the temperature scale from 14 to $673 \mathrm{~K}$ according to IPTS-68. ${ }^{7}$ Although our thermometer is not strain free, the data for the entire temperature range from 14 to $320 \mathrm{~K}$ were fitted to a single function with 21 parameters as described in IPTS-68. ${ }^{7}$ This yields a temperature scale that may have some smooth errors, but should have no "bumps." The error in the CTM heat capacity due to the temperature scale is hard to estimate since it is the derivative of the temperature scale error. Since the calibration data were originally accurate to $1 \mathrm{mK}$ and a long term shift due to strain on the platinum wire is likely to give only a uniform shift to the temperature scale, the thermometry errors should bc insignificant. In any case, improving this technique would require a much larger strain-free thermometer. This solution is impractical because of the added heat capacity and longer thermal response time.

Typically, the calibration of the thermometers is accurate to less than $1 \mathrm{mK}$ once cold, but can shift $10 \mathrm{mK}$ upon thermal cycling to room temperature. ${ }^{8}$ Therefore, it is necessary to retake the background heat capacity measurement every time the apparatus is warmed to room temperature. The cell platinum thermometer resistance is measured with an automated programmable ratiotransformer bridge that is linear to 1 part in $10^{6}$ and has less than $1 \mathrm{nV} / \sqrt{\mathrm{Hz}}$ noise.

The fractional error in the film heat capacity due to the CTM heat capacity error is simply $\delta C_{1} / C_{1}$. Because of the large background signal, we can only determine the heat capacity of a one layer film to about $1 \%$. Therefore, we only need to know the CTM heat capacity to better than $1 \%$, provided that it is stable to $0.01 \%$ between the background and sample runs to allow an accurate background subtraction.

\section{B. Heater power control and measurement}

For $0.01 \%$ accuracy in the background heat capacity, we need to know the cell heater power to that accuracy. Since the power in the electrical heater on the cell is given by $P=V^{2} / R$, we need to makc the heater voltage measurement, $V$, to within $0.005 \%$ and the heater resistance measurement to within $0.01 \%$. To meet this goal, we have constructed a heater power source that continuously monitors the heater resistance while applying the desired voltage to the heater. The circuit is based on a 16 bit D/A converter $^{17}$ that is linear to $0.00076 \%$. This gives us $0.005 \%$ accuracy for outputs that are $15 \%$ of full scale, and allows full accuracy over a wide dynamic range.

\section{Errors due to cell and CTM temperature measurement and control}

The contribution to the heat capacity due to the temperature control error is given by $G$. The main error contribution to this term is in the error in measuring $d \delta T / d t$, since $W_{1}$ and $W_{0}$ are known to $0.02 \%$ and $C_{1}$ is stable to better than $0.01 \%$. It can be shown that for normally distributed random noise on a slowly varying signal, the best way to compute the derivative is to fit the data to a line by minimizing the sum of the squared deviations. ${ }^{9}$ In this case, the noise in the derivative is $n=e 2 \sqrt{3} t^{-3 / 2}$, where $e$ is the noise per $\sqrt{\mathrm{Hz}}$ in the input data, and $t$ is the measurement time in seconds. It is assumed that there are many points in the interval. To estimate the thermometry noise required for $0.01 \%$ error, we will choose reasonable values for the parameters, and assume $W_{1}=D C_{0}$. The cell heat capacity is about $15 \mathrm{~J} / \mathrm{K}$, and the drift rate is $2 \mathrm{~K} / \mathrm{h}$. We expect the cell to come to thermal equilibrium in approximately $90 \mathrm{~s}$, so we should allow $180 \mathrm{~s}$ or longer to compute each derivative point. Then the necessary thermometry noise is $40 \mu \mathrm{K} / \sqrt{\mathrm{Hz}}$. The nominal sensitivity of platinum resistance thermometers is $40 \mu \mathrm{V} / \mathrm{K}$ using sensor currents of $0.01 \mathrm{~mA}$, so the desired noise in the resistance bridge is 
$1.6 \mathrm{nV} / \sqrt{\mathrm{Hz}}$. This is well within the Johnson noise limit for the thermometer, and a transformer coupled bridge can typically give noise performance below $1 \mathrm{nV} / \sqrt{\mathrm{Hz}}$. We use a 4 wire ac kelvin resistance bridge with two ratio transformer voltage dividers to measure $\delta T$. Its voltage noise is about $2 \mathrm{nV} / \sqrt{\mathrm{Hz}}$.

\section{Stray heat leaks and thermal design}

$L$ is the heat capacity error due to stray heat leaks. Stray heat flow into the sample must be less than or known to within $0.01 \%$ of $D C_{0}$ and stray heat flow into the CTM must be less than or known to within $0.01 \%$ of $D C_{1} W_{1} /$ $W_{0}$. If $C_{0} \approx C_{1}, D=2 \mathrm{~K} / \mathrm{h}$, and $C_{0}=15 \mathrm{~J} / \mathrm{K}$, then the stray heat leaks should be less than or known to within $0.8 \mu \mathrm{W}$. The sources of heat transfer are heat flow through the mechanical connections, thermal radiation, and gaseous conduction between the radiation shield and the bodies.

The heat conduction through the stainless steel gas fill line is caused by simple linear heat conduction. To give adequate pumping speed for eliminating contaminants from the sample cell, we decided to use a large, 1/4 in. diam fill tube. A 0.005 in. thick wall was used for mechanical stability, given a thermal conductivity of $0.5 \mathrm{~mW} / \mathrm{K}$ for a $5 \mathrm{~cm}$ length. The $28,20 \mathrm{~cm}$ long, 37 gauge copper electrical leads connected to the CTM provide $0.6 \mathrm{~mW} / \mathrm{K}$ of additional thermal conductivity. Thus, to limit the heat flow to below $0.8 \mu \mathrm{W}$, we must control the temperature of the stage above the CTM to better than $1.3 \mathrm{mK}$. If the thermal connections between the sample and the environment and between the CTM and the environment are matched to within $10 \%$, then we can allow $13 \mathrm{mK}$ error in the temperature and still achieve $0.01 \%$ error in the heat capacity ratio between the two bodies if $C_{1} \sim C_{0}$. Since it is possible to control temperatures to within $2 \mathrm{mK}$ using platinum or silicon diode thermometers, heat transfer through the fill tubes may be controlled adequately.

A temperature controlled radiation shield must enclose the bodies to minimize heat flow due to radiation. The probe must be evacuated to isolate the bodies from the radiation shields. The amount of thermal radiation can be calculated by assuming the two surfaces are parallel and of equal area, and that $\Delta T$ is small compared to $T$ (Ref. 10, page 137). To keep this heat flow below $0.8 \mu \mathrm{W}$ at $100 \mathrm{~K}$, the temperature control errors should be less than $12 \mathrm{mK}$ for typical conditions. This is a reasonable requirement for the inexpensive and easy to interface diode thermometers.

It is possible to estimate the vacuum necessary to keep the heat transfer, due to gaseous heat conduction between the radiation shield and the cell below that from thermal radiation. Helium gas is used for cooling down the cell, so we must assume that this is primary residual gas. The heat flow through helium gas is given by (Ref. 10, page 129) $2.1 a_{0} P \delta T$, where the result is in $\mathrm{W} / \mathrm{m}^{2}, a_{0}$ is the accommodation coefficient (near $1 / 2$ ), and $P$ is in Pascals. This equation takes the thermomolecular correction into account. It is assumed that the pressure is low enough for the mean free path to be greater than the $\sim 1 \mathrm{~cm}$ spacing between the radiation shield and sample cell. Then to give heat flow less than $0.8 \mu \mathrm{W}$, with $\Delta T=12 \mathrm{mK}$, the pressure should be less than $3 \times 10^{-5}$ Torr. We can easily achieve pressures less than $2 \times 10^{-6}$ Torr.

To maintain. the primary radiation shield as an equithermal surface, it is necessary to add another secondary radiation shield around it. Variations in heat flow along the surface caused by variations of emissivity, geometry, and bath temperature may cause irreproducible thermal gradients in the secondary shield.

\section{DESIGN IMPLEMENTATION}

We have argued that it is possible to achieve calorimetry that is accurate to $0.01 \%$ using a scanning technique with two nearly identical bodies, a sample and a standard. However, a practical problem stood in the way of implementing this design. To make the film heat capacity large compared with the heat capacity of the cell walls, the sample cell must have reasonably large dimensions, about 1.25 in. wide and 5 in. long. Two identical cells placed side by side and surrounded with double radiation shields would not fit in the 4 in. dewar available for this experiment. Further, two cells positioned one above the other would have made the probe too long. The solution implemented was to give up matching cells and make the CTM of a heavy block of solid copper mounted on the fill tube directly above the sample cell. In this scheme, the high CTM heat capacity reduces the temperature control requirement for the fill tube support. However, because there is no longer a near cancellation of stray heat inputs between the cell and CTM, we must increase the accuracy of the radiation shield temperature control.

\section{A. Cryogenic probe}

Figure 3 shows a schematic diagram of the calorimeter probe. During operation, the CTM receives a constant heat input from heater $\mathrm{H} 1 . \delta T$, read from thermometers $\mathrm{T} 2$ and $\mathrm{T} 1$, is input to a digital proportional-integral-derivative (PID) feedback control algorithm that controls the power to the sample cell heater HO. Variations in $\delta T$ are typically below $0.001 \mathrm{~K}$, even with rapid changes in the sample heat capacity. T0 is a platinum thermometer used to measure the absolute temperature of the sample cell. Silicon diode thermometers are located on the CTM, the isolation thermal mass, the upper thermal platform, and both radiation shields. The signals from these thermometers control the relative temperatures of these bodies to within $0.5 \mathrm{mK}$ of their set points, to prevent irreproducible stray heat flow to the CTM or sample cell. During operation, the upper thermal platform and the isolation thermal mass set points are $0.1 \mathrm{~K}$ warmer than the sample cell to eliminate the condensation of bulk solid or liquid inside the gas fill line. The heat flow coming from room temperature via the fill tube and electrical leads travels through the upper thermal platform and the helium gas heat switch, then to the liquid nitrogen or helium bath. By varying the pressure of the gas in the heat switch, we can operate either close to the bath temperature or far above it without wasting the cryogenic bath surrounding the probe. Radiation baffle $\mathrm{C}$ and the inner radiation shield enclose the sample cell and CTM in 


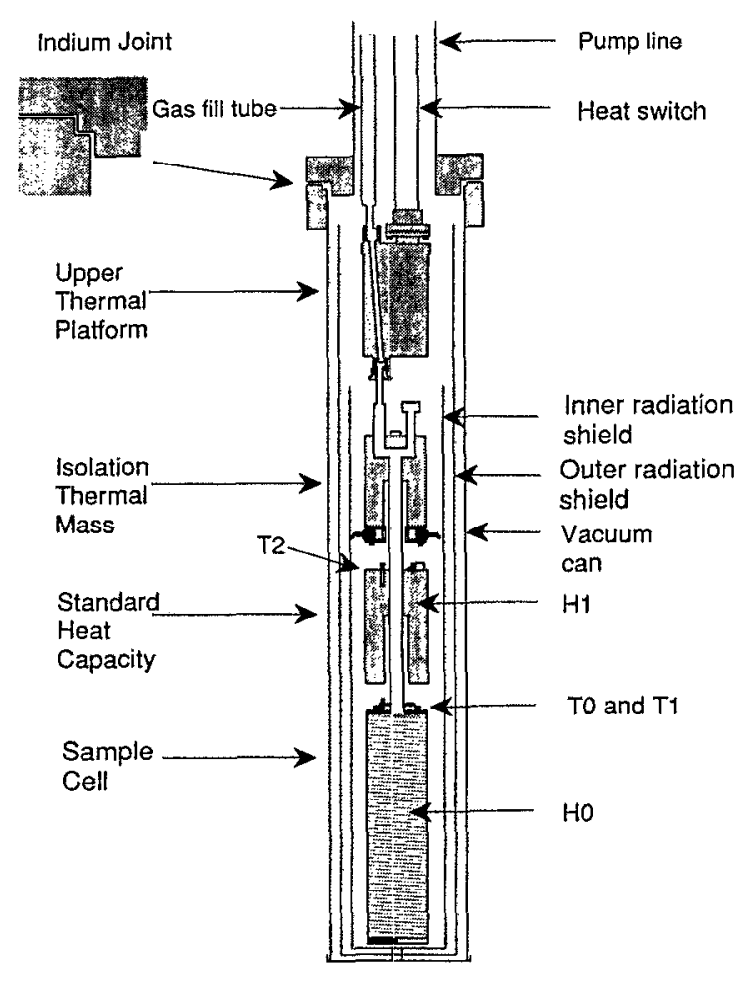

FIG. 3. Calorimeter schematic detail. T0 is the absolute platinum resistance thermometer (PRT). T1 and T2 are the two PRTs making up the differential thermometer. $\mathrm{HO}$ and $\mathrm{HI}$ are the cell and CTM heaters.

a reproducible thermal radiation environment. The hot thermal radiation from room temperature is blocked by the outer radiation shield and radiation baffles in order to allow accurate temperature control of the isolation thermal mass and the inner radiation shield. The cryogenic valve in the gas fill line may be closed to limit the volume of gas in the vapor phase in the system. Then the amount of sample gas adsorbed is more constant as the cell temperature and gas vapor pressure rise during calorimetry. The aluminum sample cell contains $28.9 \mathrm{~g}$ of Union Carbide Grafoil Mat ${ }^{11}$ which has been outgassed at $1000^{\circ} \mathrm{C}$ and $1 \times 10^{-6}$ Torr.

This design is much like the calorimeter used by Buckingham et al. ${ }^{4}$ However, we cannot enclose the successive stages within each other for a variety of reasons. The cell is cooled down by introducing helium gas into the vacuum to increase the thermal conductivity between the cell, radiation shields, and the cryogenic bath. At all times the cell must be the coldest part of the system to prevent the gas from condensing anywhere but on the graphite. Also, there cannot be a strong thermal contact between the radiation shields and the upper stages of the calorimeter, since the radiation shields must be colder than the cell during cool down. Therefore, we need twice as many temperature regulated stages compared to Buckingham et al. and it is more difficult to prevent radiation leaks between the successive stages.

\section{B. Relative temperature measurement}

The "relative resistance bridge" measures the temperature difference between the CTM and the sample cell by

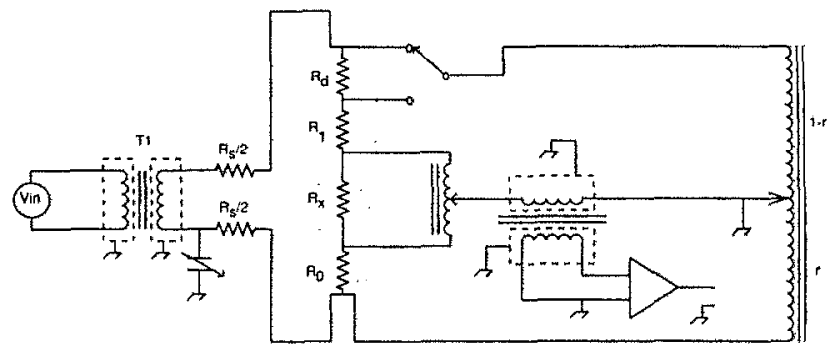

FIG. 4. Relative resistance bridge used for measuring the temperature difference between the CTM and the sample cell.

comparing the resistances of two platinum thermometers, one on the sample cell and the other on the CTM. The temperature difference must be measured with great precision, since the calorimetric accuracy depends on this measurement.

We used an ac kelvin bridge, shown in Fig. 4 that exhibits 1 to $2 \mathrm{nV} / \sqrt{\mathrm{Hz}}$ noise, repeatability better than 1 $\mathrm{ppm}$ and linearity better than $10 \mathrm{ppm}$. The thermometers, located in the cryostat, are labeled $R_{0}$ and $R_{1}$. They are connected by a short length of copper wire, resistance $R_{x}$. The circuit is grounded at the center tap of the primary ratio transformer, and the output is taken across the ground and the output of the secondary ratio transformer. The excitation is coupled to the bridge through a homemade double box shielded toroidal transformer. ${ }^{12}$

The bridge imbalance may be written as

$$
\delta R=\frac{V_{0}}{G}=R_{1}\left(T_{1}\right)-k R_{0}\left(T_{0}\right),
$$

where $k=(1-r) / r, r$ is the turns ratio, $G$ is the sensitivity, and $V_{0}$ is the output voltage. The temperature difference, $\delta T=T_{1}\left(R_{1}\right)-T_{0}\left(R_{0}\right)$, may be obtained by first calculating $R_{0}$ using the independently measured sample temperature, then deriving $R_{1}$ from the relative bridge output [Eq. (1)]. The heat capacity depends on the derivative of the temperature difference, $d \delta T / d t$, which may be obtained by differentiating the resistance imbalance directly:

$$
\frac{d \delta T}{d t}=\frac{d T_{1}}{d R_{1}} \frac{d \delta R}{d t}+\left(k \frac{d T_{1}}{d R_{1}}-\frac{d T_{0}}{d R_{0}}\right) \frac{d R_{0}}{d t} .
$$

The coefficient of the second term is about 400 times smaller than the coefficient of the first if $k \approx 1$, because the thermometer calibration curves are nearly the same.

Ideally, the detector input impedance should be much larger than $R_{0}, R_{1}$, and the output impedance of the ratio transformer, but this is not always the case for our bridge. Therefore, the bridge sensitivity is dependent on the approximate values of the sensor resistances, so it is necessary to recalculate the bridge sensitivity periodically as the temperature changes. The sensitivity must be known accurately because the temperature is measured while the bridge is off null and because the derivative of the bridge output with respect to time is a significant correction term in the heat capacity.

We implemented a unique method to calibrate the bridge sensitivity to small resistance changes. An extra 
$10.0 \mathrm{~m} \Omega$ precision resistor $\left(R_{d}\right)$ was fabricated and included in series with the thermometer on the CTM. An extra lead came out of the cryostat so that a relay could be wired to select between two configurations, with and without the extra resistance. Periodically during the bridge operation, the relay is energized for a short time. It is then possible to calculate the bridge sensitivity from the shift in the bridge output when the sensor resistance changes. The resistance value and the bridge gain were chosen to make the jump about half of the full scale output.

The signal from the bridge output transformer ${ }^{13}$ secondary is amplified and filtered with a preamplifier located in the bridge chassis before it was input to the lock-in amplifier. The control signals input to the lock ins are the reference outputs from a "frequency/phase" circuit that have been optoisolated and buffered. The computer can vary the phase of the reference signals by reprogramming the counter chips on the frequency/phase board to make one channel in phase with the bridge output, and the other $90^{\circ}$ out of phase. The lock-in outputs are digitized by voltage to frequency $(V / F)$ converters $^{14}$ whose optoisolated outputs are counted by a 16 channel 16 bit counter board that is interfaced to the metrabus ${ }^{15}$ isolated computer interface.

All the instruments use the same $V / F$ converter scheme to convert analog signals to digital and interface them with the computer. Then to isolate the different instruments from the metrabus ground level requires only two optoisolators, one for the $V / F$ output and another for the $V F C$-100's reference frequency. A standard cable and connector to all the $V / F$ outputs allow quick modification of the setup to suit the desired experiment. The $V / F$ converters are stable and linear to $0.02 \%$ in $V / F$ and $F / V$ modes. In $V / F$ mode, the converter outputs are a true integral of the input signal over the time period over which the output is counted. Thus, it is not necessary to use an antialiasing filter before the input to the converters, and the output responds quickly to changes in the input. This scheme proved an economical and convenient alternative to separate 14 bit $\mathrm{D} / \mathrm{A}$ and $\mathrm{A} / \mathrm{D}$ converters in each instrument for low speed data acquisition and control.

The metrabus ${ }^{15}$ instrument interface simply provides an external 8-bit parallel data path between the computer and each instrument, controlled by a card in the IBM PC AT. The data transfer is under complete computer control and the frequency counter and computer are synchronized by a $2 \mathrm{~Hz}$ clock that triggers an interrupt service routine in the PC. During operation, every half-second the computer reads the output from the two counter channels and records it in a file. Every 5-10 min, the PC energizes a relay that switches in the small resistor in series with the CTM differential thermometer to calibrate the sensitivity. The computer corrects the output for the ratio transformer lead wire imbalance, calculates the sensitivity, and computes the resistance imbalance in real time. The imbalance is fed into a PID control algorithm that sets the sample's heating rate.

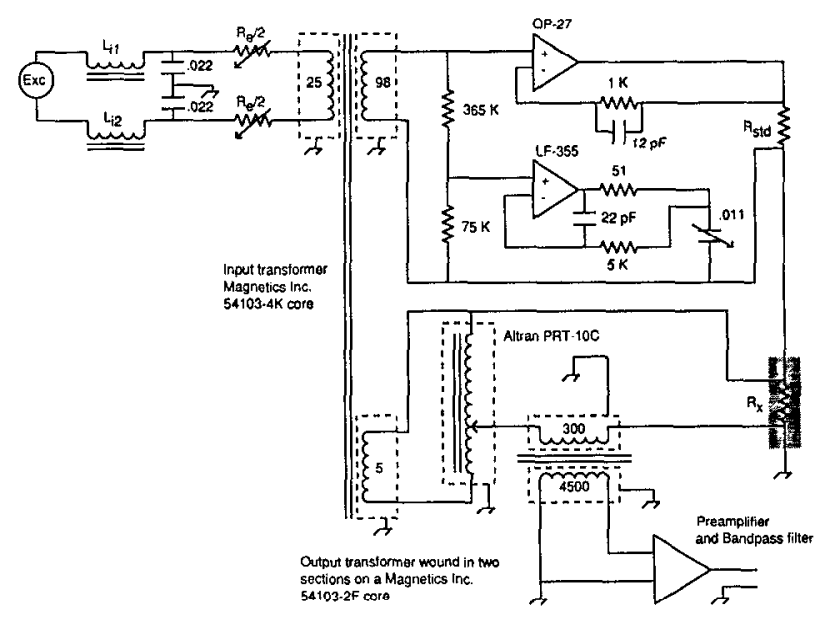

FIG. 5. Absolute resistance bridge. This circuit is a modification of one described in Ref. 16.

\section{Absolute temperature measurement}

The absolute resistance bridge used for measuring the resistance of the platinum thermometer on the cell is a modification of an active 4-wire ac resistance bridge devised by A. C. Anderson. ${ }^{16}$ This design was chosen to provide continuous automatic operation over a wide temperature range with only one programmable ratio transformer.

Figure 5 shows the circuit used for the bridge. Our goal in implementing this bridge was to make the thermometer the dominant noise source. The Johnson noise across the platinum thermometer is $0.3 \mathrm{nV} / \sqrt{\mathrm{Hz}}$ at $90 \mathrm{~K}$. Johnson noise across the standard resistor will cause a noise current to flow in the unknown, ${ }^{16} e_{\text {std }} / R_{\text {std. }}$. Then the noise voltage across the unknown is $e_{\mathrm{std}} R_{x} / R_{\mathrm{std}}$, equivalent to an extra noise resistance in series with the unknown, $R_{x}^{2} / R_{\text {std. }}$

Also, the op-amp input voltage noise $\left(e_{\mathrm{op}}\right)$ will cause a noise current through the unknown $\left(e_{\mathrm{op}} / R_{\mathrm{std}}\right)$ that causes a noise voltage across the sensor, $e_{\mathrm{op}} R_{x} / R_{\text {std }}$. If $R_{x} \sim R_{\text {std }}$, the op-amp input voltage noise will be much too large. To reduce this effect, instead of using the same number of turns in the two secondary windings of the input transformer, our resistance bridge has about 20 times more turns on the secondary that drives the current source. Then the bridge will balance when $r / 20=R_{x} / R_{\text {std }}$.

A second current source was added to provide variable amplitude current that is $90^{\circ}$ out of phase to balance the standard resistor inductance. A National LF-355 op-amp provides the current passing through the variable capacitor. Components in the feedback loop of this amplifier prevent the quadrature current source from oscillating because of the capacitive load.

To minimize the sensitivity to room temperature fluctuations, the entire circuit is mounted within a copper box within a styrofoam box inside an aluminum chassis. The copper box is heated by a temperature control circuit to regulate the temperature to $\pm 0.1 \mathrm{~K}$, so that the standard resistors remain stable to $0.5 \mathrm{ppm}$. It was discovered that 


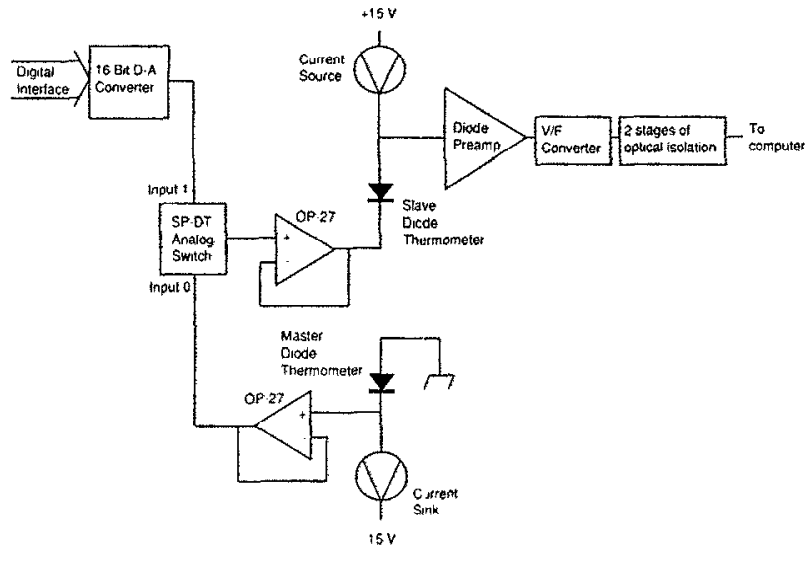

FIG. 6. Diode interface circuit for maintaining thermal isolation in the calorimeter.

two optoisolation stages were necessary to shield the bridge from high frequency noise caused by the computer. When properly grounded, there is no longer measurable interference between the computer and the bridge, the noise is below $1 \mathrm{nV} / \sqrt{\mathrm{Hz}}$ and the data are linear to about $1 \mathrm{ppm}$.

During normal operation the computer adjusts the ratiotran ratio to stay in balance. Every 5-10 min, the ratiotran ratio is changed and the output sensitivity is calculated. To find the sensitivity, the complex bridge output data are fitted to a least-squares lines calculated in real time using the data from $20 \mathrm{~s}$ before and after the ratio is changed. The bridge has operated for as long as 60 days without being reinitialized.

\section{Silicon diode thermometer interface}

Four bodies in the calorimeter provide the adiabatic environment for the sample cell and the CTM: the isolation thermal mass (ITM), upper thermal platform (UTP), and the inner and outer shields. The temperatures of the shields need to be regulated to be within about $1 \mathrm{mK}$ of the CTM temperature, and the difference between the CTM temperature and the ITM and UTP temperatures must be stable to within $1 \mathrm{mK}$. Reading the absolute temperature of a diode to within $1 \mathrm{mK}$ over a temperature range from 10 to $300 \mathrm{~K}$ requires voltage measurement accurate to within $0.0001 \%$ or 20 bits. It is much easier to measure the voltage difference between the thermometers by electrically subtracting the voltage across the diodes in the hardware and digitizing the difference.

Figure 6 shows the block diagram for the diode interface. Another diode thermometer, the "master diode" is located on the CTM and the other four diode's voltages are compared with its output. The difference voltage is amplified, digitized by the $V / F$ converter, and sent to the frequency counter board on the metrabus interface to the computer. The computer program sets the 16 bit $\mathrm{D} / \mathrm{A}$ converter output close to the negative voltage across the master diode. The master diode absolute voltage is measured with an AMP-01 instrumentation amplifier that

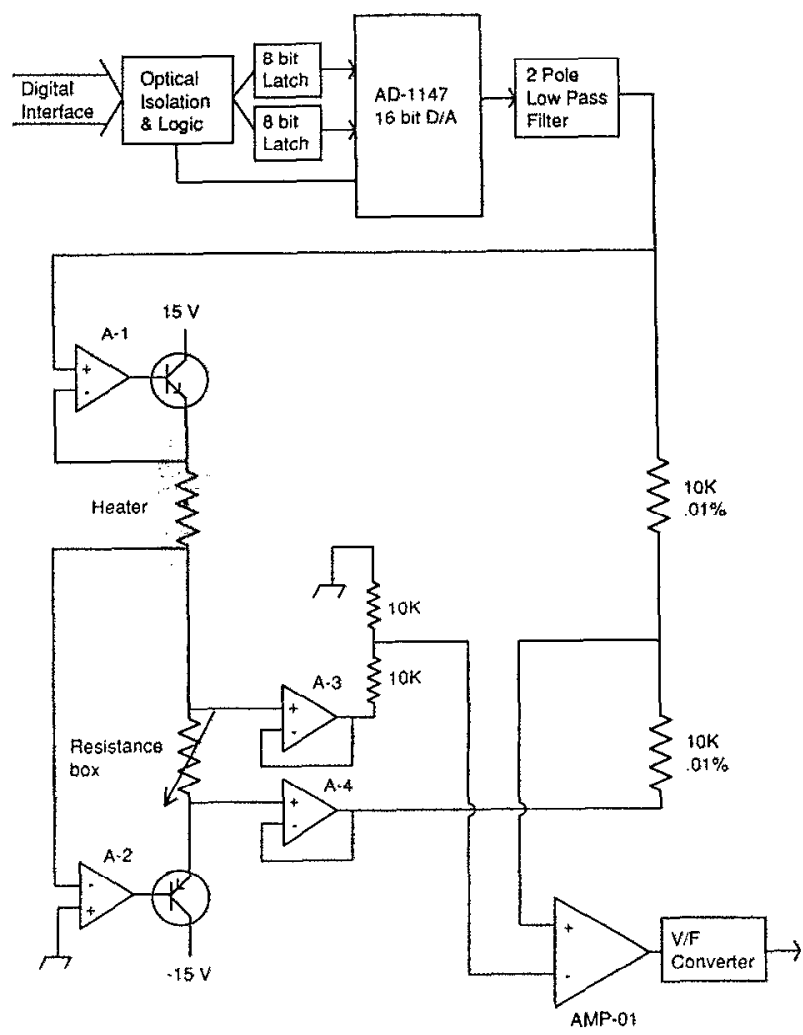

FIG. 7. Heater power circuit. The heater is one arm of a kelvin bridge so that the heater resistance may be continuously monitored.

compares the $\mathrm{D} / \mathrm{A}$ converter output to the master diode output, which is digitized by the same $V / F$ circuit as used by the slave diodes.

The computer controls the diode interface by adjusting the $\mathrm{D} / \mathrm{A}$ output. The diode interface subprogram converts the voltage to a temperature using a calibration curve (fourth-order Chebyshev polynomial over the temperature range from 65 to $120 \mathrm{~K}$ ). A large number of diodes were tested at $77 \mathrm{~K}$ to find a set of 8 that had nearly the same calibration curve. Then these were calibrated against the platinum thermometer.

The diode interface is a unique instrument that is less expensive and more accurate than commercial temperature controllers for comparing the temperatures of several bodies when they are near to each other. Although the commercial circuits may give better absolute accuracy, this circuit will provide much better isolation for the calorimeter. The noise on the relative temperature readings is about $0.3 \mathrm{mK}$ and the long term stability is about $1 \mathrm{mK}$.

\section{E. Heater power circuits}

In order to monitor the heater resistances continuously during operation, each heater is one arm of a de kelvin resistance bridge (Fig. 7). Separate, identical circuits are used for each heater. A digital interface allows the computer to set the output voltage for each $1 / 2 \mathrm{~s}$ time period. The D/A converter ${ }^{17}$ is linear to $\pm 0.00076 \%$ over a 0 - 10 $\mathrm{V}$ range and has programmable zero and full scale output adjustment. A two-pole low-pass filter smooths the voltage 


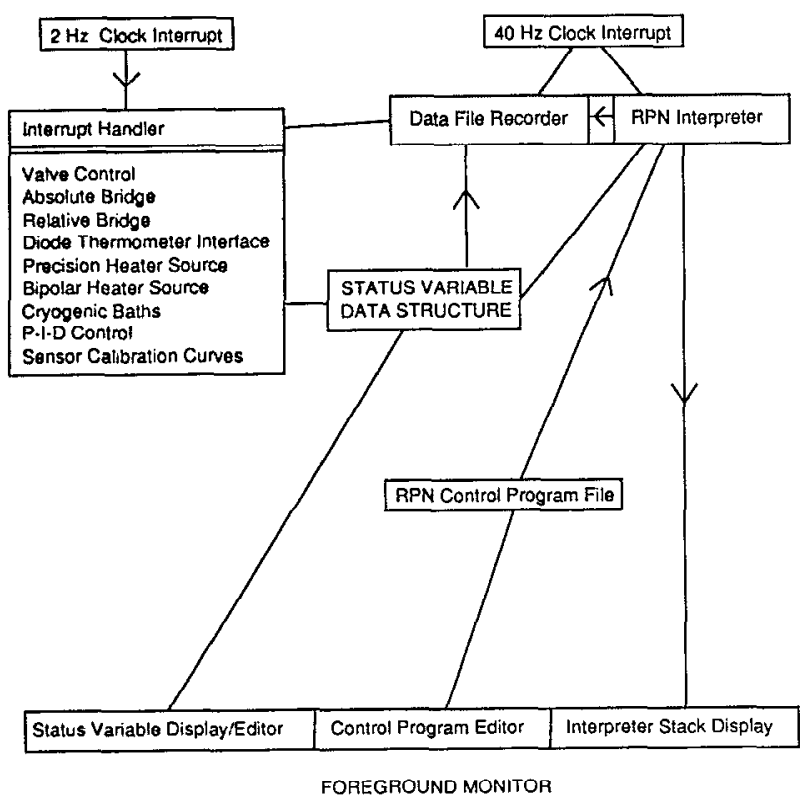

FIG. 8. Control software block diagram.

applied to the heater to prevent high frequency interference with the thermometers. The filter's response to a step input was calculated and squared to find the small correction due to the filter time constant. Software that controlled the $D / A$ converter applies this correction in real time to report the true power supplied to the heaters.

Since the power output range is fixed from 0 to $10 \mathrm{~V}$, and different experiments demand different amounts of heater power, a quad resistance heater was used for all but the upper thermal platform and fill tube heaters. Two evanohm heater wires and a single copper wire were soldered together, wound, and glued down. This forms two separate heater resistances $R_{1}, R_{2}$ that can be used singly, in series, or parallel to give four, four-wire heater resistances $\left(R_{1}, R_{2}, R_{1}+R_{2}, R_{1} \| R_{2}\right)$. Selecting the configuration with the largest resistance heater that still supplies enough power, ensures that the sample cell and CTM heater voltages are known to at least $\pm 0.005 \%$.

It is not necessary to know the exact power dissipated by the heater on the bodies providing thermal isolation to the calorimeter. For these heater channels, frequency to voltage converters are sufficient because they are linear to $0.02 \%$ and easily interfaced. The computer inputs the desired voltage to a rate multiplier ${ }^{18}$ frequency source that sends its output to a $F / V$ converter. ${ }^{14}$ A two-pole low-pass filter is used to remove the high frequency components of the $F / V$ converter output. A digitally controlled range switch chooses from $1,2,4.5$, and $10 \mathrm{~V}$ full scale output ranges to ensure that fine control is available for all circumstances.

\section{F. Computer interface and software}

An optically isolated Metrabus computer interface was chosen to interface the computer to the many digital signals from the instruments because it is very easy to connect to and allows us the construct our own instruments. Apart

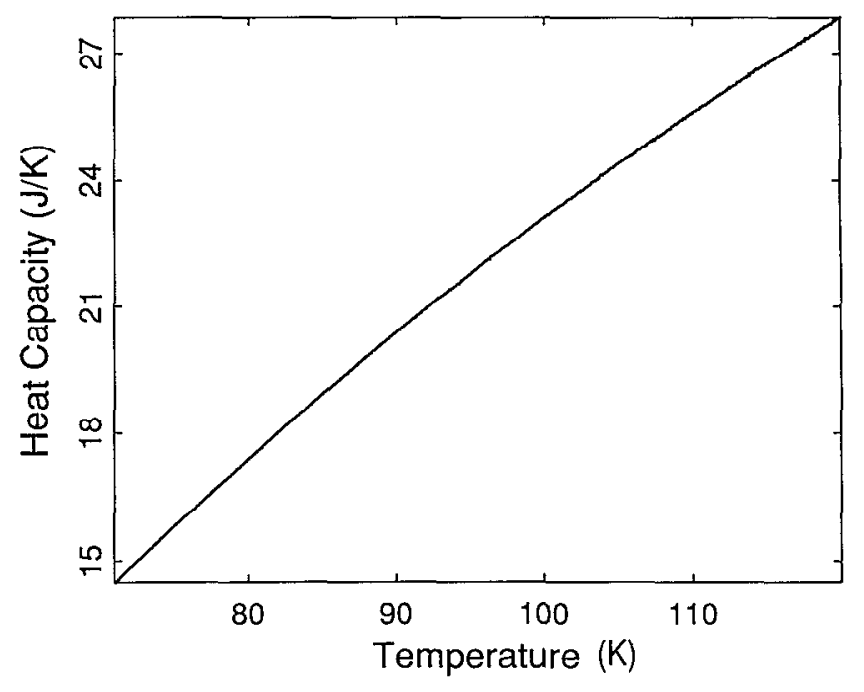

FIG. 9. Bare sample cell heat capacity in joules/kelvin.

from the instruments already described, the metrabus interfaces the computer with two room temperature thermometers, a Barocel ${ }^{19}$ pressure meter, an ion gauge controller, two cryogenic liquid level sensors, and several automatic valves for the gas handling system, the heat switch, and the cryogenic bath filling.

As with the computer interface, this experiment places unusual demands on the computer software. Instead of just controlling the higher level functions of commercial instruments, this software must also manage all the low level details of several complicated homemade instruments. For the sake of simplicity, the computer program should hide the low level details of each instrument. For efficiency, the computer code that controls each instrument should be written in a compiled computer language. Therefore, one section of the computer code is devoted to a real time compiled "C" language program that controls the operation of the instruments, making them simple to operate. Figure 8 shows the major components of the computer software. The two Hertz clock triggers the activation of a real time interrupt handler on the IBM PC. The interrupt handler runs code to control each instrument in turn using a nonpreemptive multitasking kernel. ${ }^{20}$ There are several instrument handler tasks, shown in the figure.

Each of the instrument handlers runs until it is finished performing the necessary duties for each time slice, then calls a subroutine that turns over control to the next instrument handler task. When all the tasks have finished, the interrupt handler waits until the next interrupt to activate again. The PID feedback controller has several channels that may take input from any thermometer output (optionally subtracted from any other thermometer output or fixed value) and it may be set to output to any heater channel. The Chebyshev polynomial evaluator for the sensor calibration curves, which also has several identical channels, takes any output as its input. The input is transformed using a polynomial function whose coefficients may be modified during runtime.

To control the experiment sequencing, a special pur- 


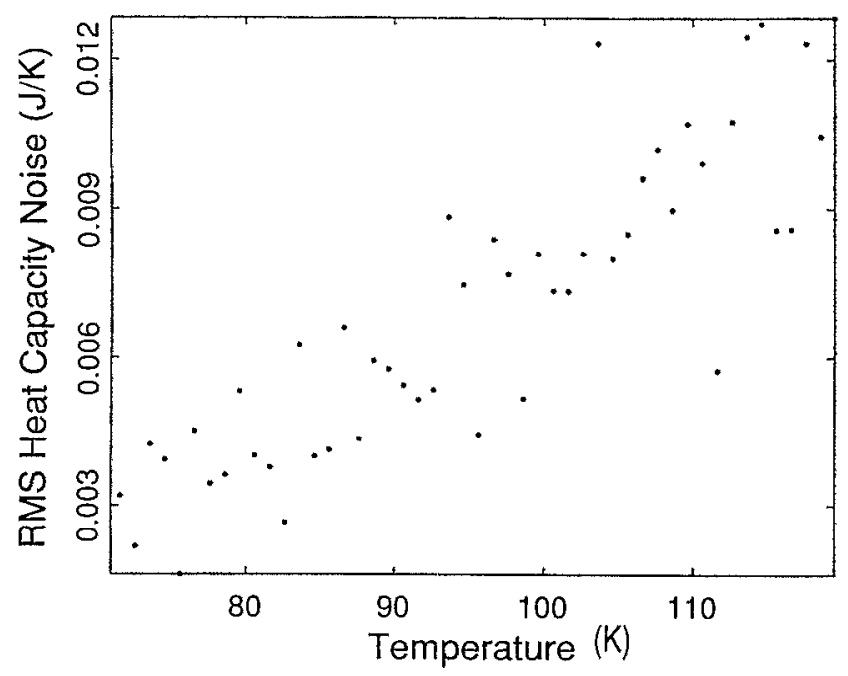

FIG. 10. Noise on the background data in joules/kelvin.

pose reverse polish notation (RPN) interpreted language interpreter was created that allows code to be changed at run time. If a compiled language were used to control higher level function, then the software that controls each instrument would need to be restarted whenever a new experiment is needed. The interpreter can perform most of the functions of a compiled language, but allows the user to change the direction of an experiment instantly.

Because the interpreter is used to setup the modes of the instruments, the interpreter can access a block of memory shared with the instrument handlers. The "status variable data structure" resides in this memory block and holds the input and output variables that are used as input and output for the instrument handlers. Interpreter commands are available that read and write to these variables in order to communicate with the instruments.

To record the data collected by the apparatus, another section of the interrupt handler calls a function within the interpreter at the end of each time slice. This function

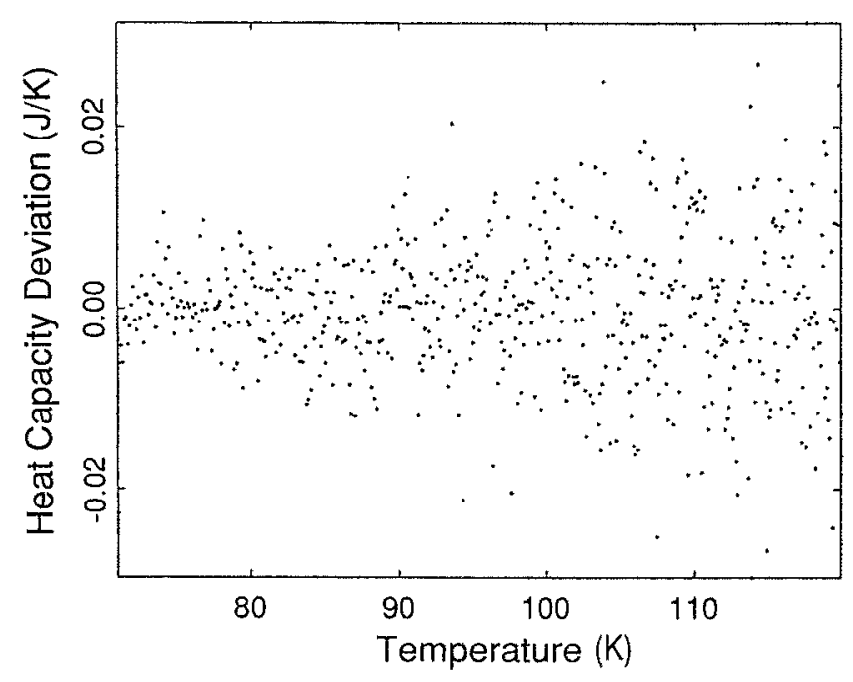

FIG. 11. RMS noise on the background data for $1 \mathrm{~K}$ intervals.

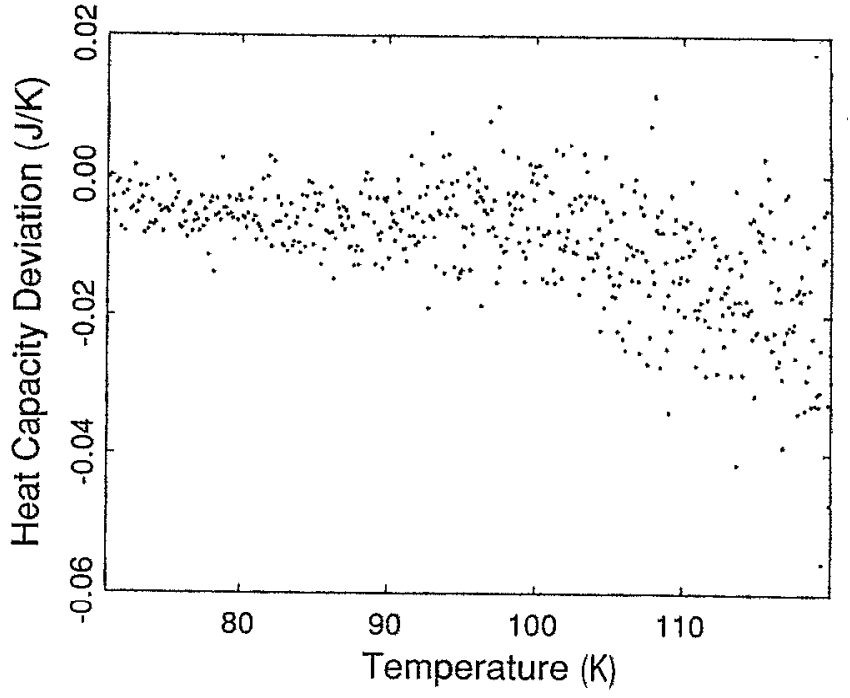

FIG. 12. Comparison of the smoothed background data from Fig. 9 with another unsmoothed background run.

writes selected variables to a buffer that the interpreter copies to a disk file. Commands run by the interpreter select which variables are to be written to the data file. Because the data collected by recording the state of the experiment every $\frac{1}{2} \mathrm{~s}$ for a $24 \mathrm{~h}$ data run can amount to more than 10 megabytes, the data are stored in binary format and it is possible to bin (average) the data from any odd number of time slices before they are recorded to the data file. Most of the data taken with the instrument is averaged over 3 time slices or $1.5 \mathrm{~s}$. Since the thermal response time of the cell is $90 \mathrm{~s}$ or longer, this has no effect on the results and greatly reduces the data file size.

Since the interrupt handler program and the RPN interpreter must constantly be running, they are loaded permanently into memory as "TSR" 21 programs. The foreground user interface program is called up from the regular DOS command line. There are three subprograms in the user interface: the RPN command file editor, the interpreter stack display, and the status variable display/ editor. A tiled windows style interface allows two of the subprograms to operate simultaneously.

\section{BACKGROUND HEAT CAPACITY SCANS, SENSITIVITY, AND REPRODUCIBILITY}

Before data could be collected, several background runs were made so that the film heat capacity could be computed. Whenever the calorimeter was warmed up to room temperature, the background calibration run was redone. The reproducibility of the calorimeter can be determined by comparing two background runs. The calorimetric precision can be found by looking at the noise on the smooth background curve. Figure 9 shows the background heat capacity of the sample cell. These data were taken at approximately $2 \mathrm{~K} / \mathrm{h}$ drift rates. The data from each background run were fitted to a third-order Chebyshev polynomial as a function of temperature to find a smoothed background. The smoothed data were subtracted from the heat capacity data to find the film heat capacity. The deviation 
of the unsmoothed background heat capacity from the smoothed data is an indication of the noise in the data, shown in Fig. 10. The rms amplitude of the deviation, computed over $0.1 \mathrm{~K}$ intervals is shown in Fig. 11. The calorimetric noise relative to the background heat capacity goes from about $\pm .015 \%$ at $72 \mathrm{~K}$ to $\pm .045 \%$ at $120 \mathrm{~K}$. This is a measure of the sensitivity of the heat capacity measurements. Figure 12 compares the smoothed background data from Fig. 9 and the data from another (unsmoothed) background run. Note that there is some systematic error in the data, but it is about as large as the random noise. Occasionally, larger noise spikes can be seen in the data. Since each point is statistically independent of the others, any feature identified by more than a few points $(2-3)$ is not due to the noise.

\section{ACKNOWLEDGMENTS}

The authors are grateful for the help of Nils Asplund who built much of this instrument. We are also indebted to Dr. Jeff Hamilton, Prof. M. W. H. Chan, Dr. G. J. Dick, Dr. D. M. Strayer, Dr. J. Delayen, Edward Boud, Dr. R. M. Housley, Dr. M. S. Pettersen, and others for their help and advice. This work was supported by DOE Grant No. DE-FG03-85ER45192.

${ }^{1}$ D. I. Goodstein, States of Matter (Prentice-Hall, Englewood Cliffs, NJ, 1975).
${ }^{2}$ M. J. Lysek, M. LaMardid, P. K. Day, and D. L. Goodstein, Langmuir 8, 898 (1992).

${ }^{3}$ P. K. Day, M. J. Lysek, M. LaMadrid, and D. L. Goodstein Phys. Rev. $B$ (to be published).

${ }^{4}$ M. J. Buckingham, C. Edwards, and J. A. Lipa, Rev. Sci. Instrum. 44, 1167 (1973).

${ }^{5}$ J. W. Loram, J. Phys. E 16, 367 (1983).

${ }^{6}$ Lake Shore Cryotronics, Inc. Westerville Ohio.

${ }^{7}$ Comité International des Ponds et Mesures, Metrologia 5, 35 (1969).

${ }^{8}$ W. V. Johnson and G. W. Lindberg, Rev. Sci. Instrum. 39, 1925 (1968).

${ }^{9}$ If the data have significant curvature, as when the calorimeter passes through a heat capacity peak, a higher order curve should be used. The heat capacity data were analysed by fitting a cubic polynomial to $\delta T$.

${ }^{10}$ G. K. White, Experimental Techniques in Low Temperature Physics, 3rd ed. (Clarendon, Oxford, 1979).

${ }^{11} \mathrm{Grafoil}$ is a registered trademark of the Union Carbide Corporation.

${ }^{12} 100$ turns primary, 200 turns secondary, on a Magnetics Inc. tape wound core, part number $54103-4 \mathrm{~K}$, each winding progressively wound in one section.

${ }^{13}$ The transformer core is made of Supermalloy, Magnetics Inc. core number 54103-2F. The primary consisted of 300 turns of two No. 21 wires in parallel, the secondary 4500 turns of No. 31 wire.

${ }^{14}$ Burr-Brown VFC-100 voltage to frequency converters.

${ }^{15}$ Metrabus is a trademark of the Metrabyte corporation.

${ }^{16}$ A. C. Anderson, Rev. Sci. Instum. 44, 1475 (1973); 47, 642 (1976).

${ }^{17}$ Analog Devices AD-1147 D to A converter.

${ }^{18}$ Standard TTL 74976 bit binary rate multiplier.

${ }^{19}$ Barocel is a trademark of the Datametrics corporation.

${ }^{20}$ The nonpreemptive multitasking kernel was a much expanded version of a multitasking system taken from: R. M. Foard, P. C. Tech. J. March, p. 53 (1986).

${ }^{21}$ A MS-DOS (T. M. Microsoft Corp.) "Terminate and Stay Resident" program is able to return control of the computer to the operating system yet retain the memory it was loaded into. Then, it can be activated by an external event such as a clock interrupt. 